sehen genoß. Die Medizinhistoriker haben dieser These gegenüber eine gewisse Skepsis bewahrt (siehe S.15 der vorliegenden Schrift, Anm.1). Sie waren dabei gut beraten, wie sich jetzt zeigt; denn Kudlien beweist erneut die Originalität des Aretaios, der zur Zeit Neros (54-68) gelebt haben muß. In einem zweiten Kapitel bringt Kudlien zahlreiche Emendationen zum griechischen Text des Aretaios, woraus sich natürlich auch neue Hypothesen für die Interpretation ergeben. Der Autor - jetzt in Kiel tätig - hat seine Studie als Mitarbeiter des Corpus Medicorum Graecorum in Berlin (Ost) begonnen und als Assistent am (inzwischen anscheinend stillschweigend stillgelegten) Medizinhistorischen Institut der Universität Würzburg abgeschlossen. Es bleibt nur zu hoffen, daß er sein Versprechen, uns eine neue deutsche Übersetzung des Aretaios zu geben, bald wahr macht!

H. Koelbing

\title{
9. Helmut Flashar, Melancholie und Melancholiker in den medizinischen Theo- rien der Antike. 145 Seiten. Walter de Gruyter, Berlin 1966. Broschiert DM 28,--.
}

Der deutsche Altphilologe Flashar hat mit dieser Monographie einen hervorragenden Beitrag zu der heute ja hoch im Kurs stehenden Geschichte der Psychiatrie geleistet, und nicht nur das: da der Begriff der Melancholie mit der antiken Säftelehre und dem Problem der psycho-physisclen Korrelationen aufs engste verflochten ist, wird unsere Kenntnis des antiken medizinischen Denkens ganz allgemein durch diese Studie erweitert und in manchem präzisiert.

Flashar zeigt zunächst, wie im Corpus Hippocraticum der Begriff der schwarzen Galle erst allmählich seine Eigenständigkeit gewinnt. Ursprünglich ist bloß von einer schwarzen Verfärbung der Galle schlechthin die Rede, erst später wird die schwarze Galle als etwas Besonderes in den Kreis der vier Kardinalsäfte aufgenommen. Ein kräftiger Drang zum Systematisieren, ein «Systemtrieb », kommt in dieser Entwicklung zum Ausdruck (S. 41). Die Viersäftelehre stammt somit nicht von Hippokrates selbst, und erst Galen hat die vier Säfte ausdrücklich den vier Elementen zugeordnet (S.108 f.; siehe auch E. Schöner, Das Viererschema in der antiken Humoralpathologie, Beiheft 4 zu Sudhoffs Arch.Gesch.Med., 1964).

Aus den sorgfältig untersuchten Einzelstellen des Corpus Hippocraticum hat Flashar ein «reiches und differenziertes Bild» von der psychischen Verfassung des Melancholikers gewonnen, das neben Angst und Trübsinn auch Zustände gesteigerter Erregung und Ekstase umfaßt (S. 46-49). Dieses Gesamtbild ist freilich aus oft weit auseinanderliegenden Elementen zusammengefügt, und es bleibt ungewiß, ob irgendeiner der hippokratischen Autoren die Melancholie je so gesehen hat. Wird hier die Sicht Flashars nicht schon etwas zu sehr von der peripatetischen Auffassung einer melancholischen Konstitution (S. 62-68) bestimmt, die mit hoher künstlerischer und intellektueller Begabung verbunden ist, aber auch zum Ausschwingen der Stimmungslage in die Extreme der Depression und der Manie disponiert und damit der Zyklothymie Kretschmers sehr nahe kommt? 
Bei Diokles hebt Flashar die Verbindung melancholischèr Verstimmung mit Verdauungsstörungen hervor (S. 51), bei der schon erwähnten Schule des Aristoteles auch die Erkenntnis erhöhter Selbstmordgefährdung, bei Aretaios die scharfe klinische Beobachtung: hier findet sich zum erstenmal die Definition der Melancholie als einer Mutlosigkeit, die auf einer einzigen Wahnvorstellung beruht (S. 77) - also das «délire exclusif» Pinels, die «monomanie» Esquirols. Soran, der die Melancholie ohne humoralpathologische Begründung darstellt und durch seelische und geistige Einflüsse zu beheben sucht, wird gebührend gewürdigt. (Der Methodiker Soran hat die Melancholie im allgemeinen auf eine zu große Spannung des Gewebes mit Verengung der hypothetischen Poren - status strictus - zurückgeführt, nicht - wie auf S. 82 irrtümlich mitgeteilt wird - auf Erschlaffung.)

Geradezu begeistert äußert sich Flashar über RuFus. «Selbst die unvollständige Kenntnis der Schrift des Rufus» - wie Flashar sie aus den überlieferten griechischen, arabischen und lateinischen Bruchstücken mit großer Umsicht für sich rekonstruiert hat - «läßt das Urteil zu, daß Galens Abhandlung über die Melancholie ... durch Rufus weit in den Schatten gestellt wird» (S.104). GaLENS Leistung liegt einmal mehr in der Systematisierung des Vorgefundenen. Damit hat er u.a. auch die mittelalterliche, bis heute nachwirkende Doktrin der vier Temperamente vorbereitet.

Besonders verdienstvoll ist es, daß Flashar auch die nachgalenischen Autoren Poseidonios (der nicht mit Ciceros Lehrer, dem Stoiker Poseidonios identifiziert werden darf, sondern erst im 4.Jahrhundert n. Chr. lebte) und Alexander von Tralleis in seine Untersuchung einbezieht. Besonders der letztere erscheint als Mann von Format, der die überlieferten Lehren selbständig durchdacht und gestaltet hat und seine Darstellung mit zahlreichen klinischen Beobachtungen untermauert.

Es ist Flashar gelungen, den antiken Melancholiebegriff in der ganzen Komplexität und Differenzierung, die die historische Entwicklung ihm verlieh, darzustellen. Seine Aussagen sind durchwegs gut belegt; vor interessanten, aber ungenügend fundierten Spekulationen hütet er sich. (Daß da und dort auch andere Interpretationen und Akzentsetzungen möglich sind, versteht sich von selbst). Zudem hält es Flashar nicht unter seiner Würde, klar und verständlich zu schreiben. All dies verleiht seiner Studie eine Zuverlässigkeit, die man leider gerade im Bereich der sogenannten Problemgeschichte nur zu oft vermißt.

H. Koelbing

10. Hans Jenzer, Dr. med. Johann Friedrich von Herrenschwand, ein Berner Arzt im 18. Jahrhundert. 266 Seiten. Francke, Bern 1967. Fr. 29.-.

Der Autor, Spezialist für innere Medizin und Privatdozent für Geschichte der Medizin in Bern, hat mit großer Sorgfalt alles, was sich in Büchern, Akten, Briefen und anderen Schriften über J.F.v. Herrenschwand (1715-1798) finden ließ, durch- 